\title{
Human Ergonomics Study in Microgravity Environment
}

\author{
Pengyan Liu ${ }^{1}$, Dong Zhou ${ }^{1}$, Long Xue ${ }^{1}$ and Yuan $\mathrm{Li}^{1}$ \\ ${ }^{1}$ Beihang University, Beijing, China
}

\begin{abstract}
In recent decades, human exploration of space has gradually deepened, and more and more countries and regions have successfully sent astronauts into space. How to complete the space experiment as efficiently and safely as possible in the shortest time with limited resources has become an important issue in the field of aerospace technology. Taking full account of such matter, we consider that ergonomics in design stage is one of the most effective ways to solve this problem. The microgravity environment is the biggest difference between ground and space. Therefore, the study of ergonomics under the microgravity environment is of great significance. This article deeply analyses and summarizes the physiological differences between human and normal gravity under microgravity environment. From the ergonomics point of view, the requirements for spacecraft design and space mission planning are put forward for the reference of engineers and scholars.
\end{abstract}

\section{Introduction}

With the improvement of aerospace science and technology, the United States, Russia, China and many other countries have successfully sent humans into space. Since the beginning of the establishment of space stations by humans, the astronauts' length of stay in space has also been continuously refreshed. To ensure the safety of astronauts and improve work efficiency, spacecraft design must fully consider ergonomics.

Ergonomics take the human-oriented principle, focusing on human physical and psychological factors, research on the relationship between people, machines and the working environment to determine the best combination [1]. Unlike the operation task in ground conditions, the astronauts are in the microgravity environment during orbital operations, and their visual, operational, cognitive, and strength are different from those in the $1 \mathrm{G}$ gravity environment. Research shows that the application of human factors engineering plays an important role in space safety, especially in space operations and the human factor [2]. On the other hand, astronauts' work in space is tough, therefore they need to complete as many experiments as possible within a limited time quickly as they can. In addition, unlike the feature that the support equipment on the ground can support personnel movement for a long time, the spacecraft transportation capacity is limited and the protection resources are very expensive. Therefore, consider ergonomics fully in the design process of spacecraft is of great significance to ensure personnel safety, shorten task time, increase task completion rate, and reduce maintenance costs.
This article first elaborates in Chapter 1 the importance of considering ergonomics in the design of spacecraft systems. Then, on the basis of large amount of literature, the results of the research on the effect of microgravity environment on human physiology, cognition and accessibility are introduced in Chapters 2, 3 , and 4 respectively. In Chapter 5, the physiology of the astronaut is summarized, and some suggestions on the design of the spacecraft and the space mission are put forward.

\section{Effects of microgravity on human physiology}

\subsection{Influence of microgravity on human body}

Long-term weightlessness will affect the size and posture of the human body. As the astronauts have been living under gravity on the ground, their body will experience a downward force. This force has always existed on the earth, and people's height generally does not change in a short time. However, under the condition of microgravity, when the body's downward gravity is lost, the spine will be lengthened, the upper body's size will also increase, and the overall height will increase by about $3 \%$.

The neutral posture under microgravity will change, with the plantar flexion of the ankle (bending towards the sole of the foot), the flexion of the hip and knee joints, slight hip abduction, slight flexion of the trunk forward, and movement of the head and limbs towards the midpoint of the range of motion, shoulders, arms, and elbows lift up and the neck bends forward, as shown in Figure $1[3-6]$. 

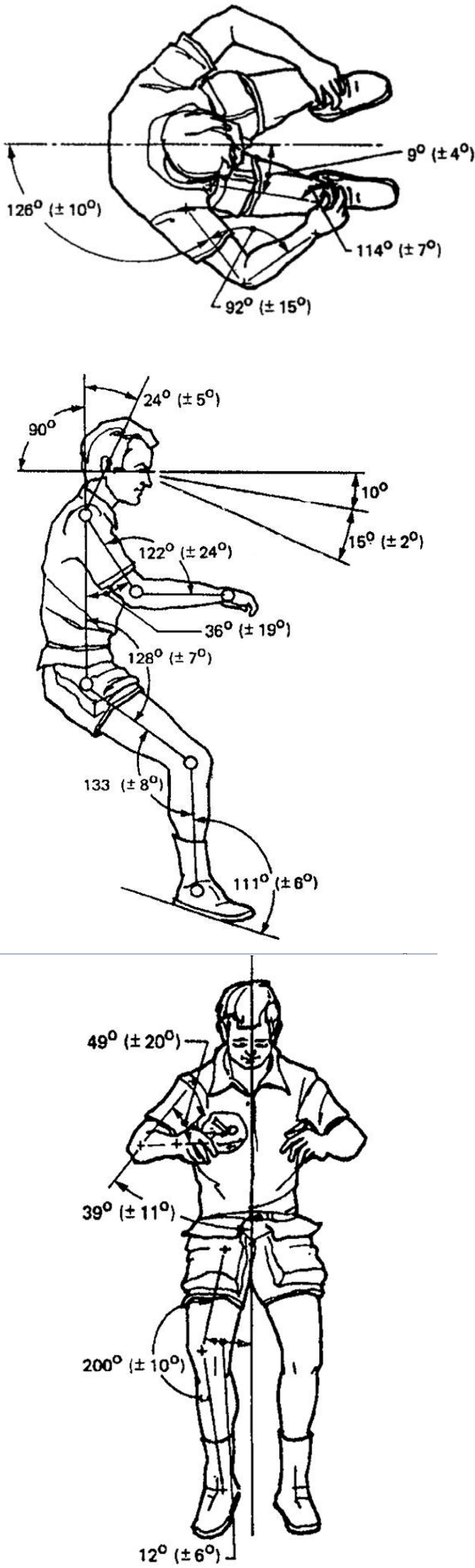

Figure 1. The neutral posture under microgravity

\subsection{The effect of microgravity on skeletal muscle}

In the microgravity environment, skeletal muscles suffer from problems such as atrophy and bone loss, resulting in decreased muscle strength. The entire center of mass of the human body changes, as well as the ability to exercise. These changes will have a great impact on the accomplishment of the astronauts' tasks in the space station, especially for work that requires strength. Biomedical research shows that the microgravity environment can lead to a decrease in the number of muscle proteins and can easily lead to functional changes such as muscle fatigue [7]. At the same time, the muscle weight loss of the lower extremities will significantly exceed the muscle weight loss of the upper extremities. Animal experiments have shown that the rat's hind-limb soleus muscle has a 23 to $40 \%$ loss in weight, whereas the forelimb muscle's weight loss is only 12 to $24 \%$ [8].

\subsection{Bone and Mineral Metabolism}

When people are in a microgravity environment for a long time, their bones will grow due to the lack of weight, and their height will increase by about $5 \mathrm{~cm}$. Loss of weight will lead to lower bone density in the lower body, and the skull bone density will increase [9]. This phenomenon is related to the tendency that body fluid concentrates on the head. Because of the change of bone metabolism, a large amount of calcium will be lost [10]. According to the data of Skylab, on-track astronauts lose $0.6 \%$ of the total calcium per month on average $[11,12]$.

\section{The influence of microgravity on cognition}

Cognitive ability is controlled by the human brain and central nervous system, including human emotions, memory functions, language, thinking and other psychological states. The microgravity environment can lead to a decline in the ability of movement, reaction, and vision which closely related to mission operations [13]. For example, the response time for sound, vision, and selection under microgravity will increase by $100 \mathrm{~ms}$, $120 \mathrm{~ms}$, and $1000 \mathrm{~ms}$, respectively. This change can lead to problems such as spatial disorientation, posture illusion and ataxia, as well as lengthening the task completion time [14].

\subsection{Spatial orientation and object recognition}

Under the microgravity condition, the downward gravitational sensation has disappeared, and thus the vestibular system that controls the body's adaptation to the earth's gravitational sensations has also changed. The most important directional sensory vestibular system is the most affected. The vestibular system under microgravity can cause eye movement disorders, and decreased ability of posture control and coordinate movement. The absence of vertical gravity and downward inertial forces will cause the misjudgement on own body position and movement illusions. The human body loses its long-term dependent gravity-referenced 
coordinate system under microgravity. Therefore, it will lead to wrong judgment of the object's distance [15].

\subsection{Motor perception}

The astronaut's cognitive mode of moving objects under microgravity will change. On the Earth, because of a force toward the center of the earth, people are used to using this downward force as the reference for motion. Under microgravity, due to the loss of the habitual coordinate system, the astronaut's vestibular system and somatosensory sensation will make mistakes when judging moving objects [16]. During the first 2-3 days of a spaceflight, $60-80 \%$ of the astronauts will be affected by space motion sickness, which is a typical motion sickness produced during adaptation of the vestibular system to the microgravity environment [17]. At the same time, due to changes in the angle of view, the ability to determine the movement of objects will be biased. The astronauts usually establish reference frames in the space station with self-centeredness, which may lead to obstacles in motor perception [18, 19].

\subsection{Coordinated movement}

Coordinated movement relies on information input from systems such as vestibular systems, somatic sensation, and vision which may work together to complete the coordinated movement of the body. When the astronauts are in a microgravity environment for a long time, these coordinated motion information control systems will undergo significant changes. Since there is no downward gravity, the control of the posture in the space station can be difficult which requires the external forces to control [20]. The correlation between different parts of the movement exercise ability and gravity are different. Among them, the movement is the biggest changed part. The astronauts are walking with their feet on the ground, while floating laterally under microgravity. The adjustment of posture is even more difficult than on the ground and requires handrails. The overall coordination ability of people will decline, and the speed of action will be affected [21].

\section{The effect of microgravity on accessibility to operation}

Microgravity can affect the visual reachability of operational accessibility. During the operation of the mission, the astronaut's line of sight must stay on the operation. If the perspective is affected, the task will be delayed and the corresponding action will be slow. Under the condition of microgravity, the human body is completely relaxed.

Under the condition of $1 \mathrm{G}$, the best viewing zone of the upper and lower viewing zones is located at $15^{\circ}-45^{\circ}$ below the horizontal line of sight, and the effective viewing zone is located at $0^{\circ}-75^{\circ}$ below the horizontal line of sight, as shown in FIG. 2 . Under the microgravity condition, the layout design should be based on the change of the main line of sight falling by $15^{\circ}$ in the neutral posture state, as shown in FIG. 3. Regardless of $1 \mathrm{G}$ conditions or under weightless conditions, the best view zone for the left and right viewing zones is the left and right $15^{\circ}$ viewing zone, and the effective viewing zone is the left and right $95^{\circ}$ viewing zone.

The perspective of the operation determines whether the action can be carried out normally, because the astronaut needs to focus when conducting the action and stay focused [22]. As microgravity changes the astronaut's normal physiology perspective, and visibility is an important factor in the human-computer interaction process, changes in the perspective of the activity will cause difficulties for the astronauts and increase the task completion time and reduce work efficiency.

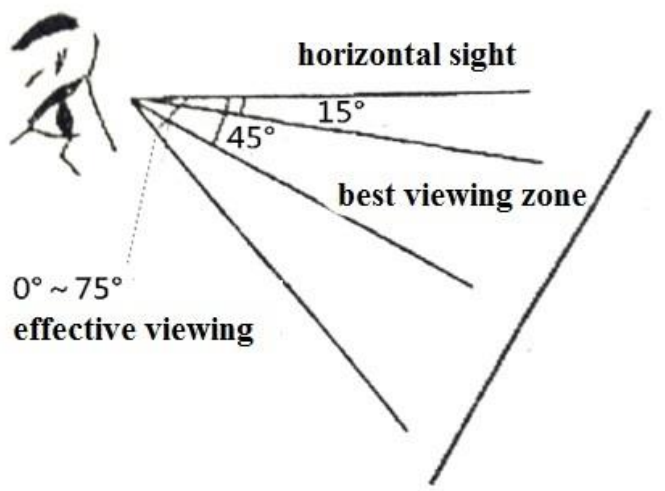

Figure 2. The upper and lower view in $1 \mathrm{G}$ condition

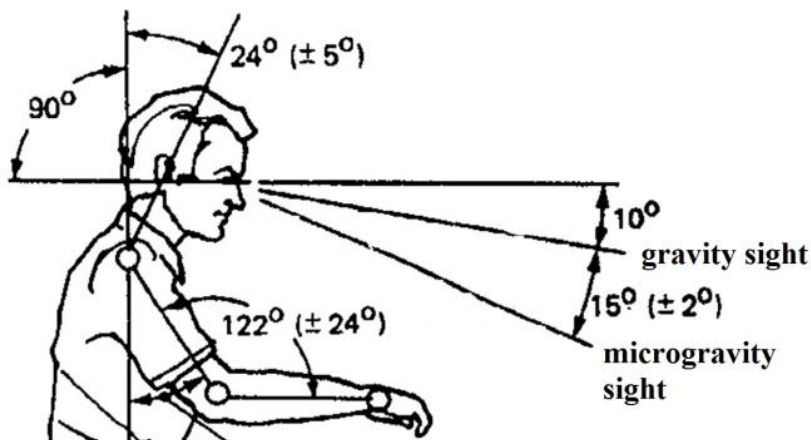

Figure 3. Changes in the upper and lower viewing zones in a microgravity environment

\section{Conclusion}

This article mainly summarizes the influence of microgravity environment on the astronaut's physiology, cognition and accessibility. Astronauts are the main factor of space activities, and changes in astronauts' physiology affects the completion of space missions. For example, the changes of cognition will affect the astronauts' decisions and prolong the reaction time, thereby increasing the difficulty of the task and the duration of the task. The influence of visibility puts forward new design requirements for the placement of experimental instruments and operating interfaces. 


\subsection{Influence of Microgravity on Human Physiology}

The limitations of astronauts under microgravity are mainly reflected in the following aspects:

(1) The decline in muscle power;

(2) People's various types of information processing mechanisms, such as tactile, pain, visual, etc., receive slower responses;

(3) Decreased spatial orientation of the human body;

(4) The person's movement speed slows down and depends on assistance;

(5) Changes in the human field of vision and optimal operating space;

(6) The overall judgment ability is reduced.

\subsection{Ergonomics Requirements in Microgravity Environment}

Based on the physiological changes of humans in the microgravity environment analyzed in the previous article, the space environment poses the following requirements for the ergonomic design of spacecraft:

(1) Minimize work that requires long-term execution and greater strength;

(2) Minimize work that requires long-term attention and work that cannot be suspended halfway;

(3) Increase the positioning and orientation signs in the spacecraft to help the astronauts carry out spatial orientation;

(4) Set foot limiters, hip limiters and handrails with suitable position, size, and quantity to assist astronaut activities and operating facilities;

(5) Reasonably arrange the layout of each component and laboratory equipment in the design process in order to be consistent with the astronauts' vision and comfortable operation space in the microgravity environment;

(6) Minimize the occurrence of multitasking in a short period of time;

(7) Set up a reasonable emergency plan for emergencies to avoid astronauts to judge by themself, so as to reduce the probability of error occurrence.

\subsection{Conclusion}

This paper analyzes the influence of the microgravity environment on the astronauts' physiology and operational activities, and obtains the differences from the tasks performed on the ground in the $1 \mathrm{G}$ environment. It provides suggestions and references for ergonomics design of spacecraft and space station in microgravity environment.

\section{References}

1. M. Oshima, Ergonomics, Iyo Denshi to Seitai Kogaku Japanese Journal of Medical Electronics \& Biological Engineering, 10.6:556 (1972)

2. A. R. Brody, Space operations and the human factor, Aerosp Am, 31.10:18-21(1993)
3. V. V. Bogomolov, A.I. Grigoriev, I.B. Kozlovskaya, The Russian experience in medical care and health maintenance of the International Space Station crews, Acta Astronautica, 60(4-7):237-246 (2007)

4. S. Glasauer, H. Mittelstaedt, Perception of spatial orientation in microgravity, Brain Research Reviews, 28(1-2):185-193 (1998)

5. G. Leone, The effect of gravity on human recognition of disoriented objects, Brain Research Brain Research Reviews, 28(1-2):203-214 (1998)

6. P. P. Di, M. V. Narici, Muscles in microgravity: from fibres to human motion, Journal of Biomechanics, 36(3), 403-412, (2003)

7. G.B. Prange, L.A. Kallenberg, M.J. Jannink, et al, Influence of gravity compensation on muscle activity during reach and retrieval in healthy elderly, $J$ Electromyogr Kinesiol, 19(2):e40-e49 (2009)

8. A. Ishihara, F. Nagatomo, M. Terada, et al, Effects of microgravity on the mouse triceps brachii muscle, Muscle \& Nerve, 52(1):63-68, (2015).

9. P. Ghosh, J. N. Stabley, B.J. Behnke, et al, Effects of spaceflight on the murine mandible: Possible factors mediating skeletal changes in non-weight bearing bones of the head, Bone, 83:156-161, (2016).

10. S. M. Smith, M. Heer, L. C. Shackelford, et al, Bone metabolism and renal stone risk during international space station missions, Bone, 81:712-720, (2015).

11. M.F. Reschke, J.J. Bloomberg, D.L.Harm D L, et al, Posture, locomotion, spatial orientation, and motion sickness as a function of space flight, Brain Research Brain Research Reviews, 28(1-2):102-117 (1998)

12. J. Wei, The hierarchical characteristics of physiological effects of weightlessness, Aerospace Medicine and Medical Engineering, (2):134-139, (1994), (in chinese)

13. K. Koga, Gravity cue has implicit effects on human behaviour, Aviat Space Environ Med, 71(9):78-86. (2000)

14. J. Mcintyre, A. Berthoz, F. Lacquaniti, Reference frames and internal models for visuo-manual coordination: what can we learn from microgravity experiments, Brain Research Brain Research Reviews, 28(1-2):143 (1998)

15. T. Pozzo, C. Papaxanthis, P. Stapley, A Berthoz, The sensorimotor and cognitive integration of gravity, Brain Research Reviews, 28(1-2):92-101 (1998)

16. G.D. Gironimo, C.D. Martino, A. Lanzotti, et al. Improving MTM-UAS to predetermine automotive maintenance times, International Journal on Interactive Design \& Manufacturing, 6(4):265-273 (2012)

17. W. Chen, J. G. Chao, J. K. Wang, et al., Subjective Vertical Conflict Theory and Space Motion Sickness, Aerospace Medicine \& Human Performance, 87(2), (2016).

18. J.R. Lackner, P. Dizio, Motor function in microgravity: movement in weightlessness, Current Opinion in Neurobiology, 6(6):744-750 (1996)

19. D. Manzey, B. Lorenz, Mental performance during short-term and long-term spaceflight, Brain Research Reviews, 28(1-2):215-221 (1998) 
20. B. Yang, T. Hu, Research and analysis of space station microgravity environment, Manned Space Flight, (2):178-183 (2014), (in chinese)

21. B. Fowler, D. Manzey, Summary of research issues in monitoring of mental and perceptual-motor performance and stress in space, Aviation Space \& Environmental Medicine, 71(9 Suppl):76-7.a (2000)

22. G. Clément, Alteration of eye movements and motion perception in microgravity, Brain Research Reviews, 28(1-2):161-172 (1998) 